\title{
Quantum Mysteries for No One
}

\author{
Frank Lad \\ Department of Mathematics and Statistics, University of Canterbury, Otautahi/Christchurch, New Zealand \\ Email: frank.lad@canterbury.ac.nz
}

How to cite this paper: Lad, F. (2021) Quantum Mysteries for No One. Journal of Modern Physics, 12, 1366-1399. https://doi.org/10.4236/jmp.2021.129082

Received: May 14, 2021

Accepted: July 23, 2021

Published: July 26, 2021

Copyright $\odot 2021$ by author(s) and Scientific Research Publishing Inc. This work is licensed under the Creative Commons Attribution International License (CC BY 4.0).

http://creativecommons.org/licenses/by/4.0/

\begin{abstract}
I provide a critical reassessment of David Mermin's influential and misleading parable, "Quantum Mysteries for Anyone", identifying its errors and resolving them with a complete analysis of the quantum experiment it is meant to portray. Accessible to popular readership and requiring no knowledge of quantum physics at all, his exposition describes the curious behaviour of a machine that is designed to parody the empirical results of quantum experiments monitoring the spins of a pair of electrons under various conditions. The mysteries are said to unfold from contradictory results produced by a signal process that is proposed to explain them. I find that these results derive from a mathematical error of neglect, coupled with a confusion of two distinct types of experiments under consideration. One of these, a gedankenexperiment, provides the context in which the fabled defiance of Bell's inequality is thought to emerge. The errors are corrected by the recognition of functional relations embedded within the experimental conditions that have been long unnoticed. A Monte Carlo simulation of results in accord with the actual abstemious claims of quantum theory supports probability values that Mermin decries as unwarranted. However, the distribution it suggests is not definitive, in accord with the expressed agnostic position of quantum theory regarding measurements that cannot be executed. Bounding quantum probabilities are computed for the results of the gedankenexperiment relevant to Bell's inequality which inspired the parable. The problem is embedded in a 3 $\times 3$ design of Stern-Gerlach magnet orientations at two observation stations. Computational resolution on the basis of Bruno de Finetti's fundamental theorem of probability requires the evaluation of a battery of three paired linear programming problems. Though technicalities are ornate, the message is clear. There are no mysteries of quantum mechanics that derive from mistaken understandings of Bell's inequality... for anyone.
\end{abstract}

\section{Keywords}

Bell Inequality Violation, Fundamental Theorem of Probability, Local Realism, Probability Bounds 


\section{Reassessing the Quantum Mysteries of David Mermin}

Detailed mathematical formalities of theoretical quantum mechanics preclude their understanding by even the technically sophisticated among the generally educated public. Replete with measurement operator matrices on a Hilbert space of quantum states and a peculiar style of notation that is unique to them, engagement with their prescriptions is forbidding. Aware of the widespread public interest in the inscrutable content of the theory, David Mermin [1] devised an engagingly simple parable to provide an exhibition of touted features of mysterious quantum behaviour as they have been long understood. Requiring no knowledge of any aspect of quantum physics at all, the exposition merely describes a machine that sends a pair of balls in opposite directions from a central station $C$ to detectors at stations $A$ and $B$. The balls can address each detector in three different ways, represented by three numbered settings of a dial on its face. Thus, there are nine different conditions under which an experimental run of the machine can be conducted. Coloured lights, either red or green, at the two detectors, provide signals as to what occurs in the encounters of the balls at the two stations. Statistical properties of the signal performance in a sequence of operations of the device are reported and explained in such a way as to exhibit one of the defining puzzling mysteries of quantum theory: the purported defiance of Bell's inequality by the probabilistic behaviour of entangled particles.

Questions arise concerning the physical process of production of the machine's output. This evidently involves entangled probabilities of light signals at the stations $A$ and $B$, each of which depends on both the dial setting at its own station and the setting at the other station. This is despite the fact that there is no physical connection between the stations which might convey information between them regarding their respective dial settings. An information transmission scheme is envisioned by which the pair of balls may carry within themselves unobserved encoded messages to stimulate the observed entangled behaviour of the light signals. Although this is shown capable of accounting for regularly matching signals when the dial settings are identical, an enigma arises when the settings are different. Any such scheme appears to instigate matching light signals at the two stations with a frequency exceeding $1 / 3$ in situations for which the machine is known to exhibit such signalling with probability of only $1 / 4$. The machine behaviour is touted as mysterious, defying explanation by encoded messages and portraying one of the great mysteries of quantum analysis.

Upon completion of the exhibition, it is explained to any QM-enlightened readership that the parable of the mysteries actually mimics the situation of a real quantum experiment. This would involve the transmission of a pair of electrons in opposite directions over long distances toward two observation stations at which Stern-Gerlach magnets identify the electromagnetic spin of each electron as directed up or down. The magnets at the two stations can each be set up in any of three differently angled directions perpendicular to the direction of the incoming electrons. These alternative directions are represented in the parable by the three different settings of the dials at stations $A$ and $B$. The statistics re- 
ported in the parable summarizing signal behaviour of the machine over sequences of experiments at each dial pairing correspond to what is expected of the spin observations according to the principles of quantum theory.

The reported results are both simple and stunning. The professor teased that he could actually create this machine using the results of paired quantum experiments as the generators of the random outcome sequences. Requiring an effort which he assured would be somewhat less than the order of the Manhattan project, he proclaimed that "the conundrum posed by the behavior of the device is no mere analogy, but the atomic world itself, acting at its most perverse."

So engaging, simple, and startling is Mermin's exhibition that the piece has become standard fare for the exposition of Bell's inequality to students ever since, both students of physics and of philosophy, even at graduate levels. Moreover, it is included in a welcome and popular collection of his essays on matters of theoretical physics meant for the generally educated public, Boojums all the way through: communicating science in a prosaic age. Immensely successful and influential, it has been reprinted by now in nine hardcover and five paperback editions. The exposition of "Quantum Mysteries" was lauded by Richard Feynman as "one of the most beautiful papers in physics that I know of" according to the preface to the volume [2].

However, the lionization of Mermin's article by another leading figure of twentieth century physics does not make it correct. I make bold here to display that his amusing allegorical presentation of the situation is both mistaken and misleading. A recognition of my assessment here, in tandem with my arguments in [3] and [4] will suggest a revision of physicists' attitudes towards the interpretation of quantum theory, and the mistaken supposed defiance of Bell's inequality in particular.

David Mermin is one of the most accomplished physicists of our era, a cherished professor in the Department of Physics at Cornell University for many years. It is edifying to view his curriculum vitae at the public website at cornell.edu. Along with the publication of his own extensive research results, he has been seriously committed to the exposition of contemporary findings of theoretical and applied physics to the general public. In addition to 138 technical publications, his curriculum vitae includes 20 pedagogical articles and 29 general writings. This does not make him immune to mistakes. We all make mistakes. It is with due respect for his accomplishments and appreciation of his personal style that I explain in this article the serious consequences of his mistaken understanding of Bell's theorem and its implications. Feeling gauche to refer to him regularly throughout the essay as "Mermin", I refer to him alternately as "the professor". I intend this with respect.

Of course I invite you to read or to reread the parable of "Quantum Mysteries for Anyone" for yourself, along with the preface to Boojums as a prelude to studying my exposition. Both are available online. However, to make this presentation self-contained I will begin Section 2 with a faithful outline of the mysteries as they are portrayed, firstly with a description of the properties of the ma- 
chine's operation, and then with a display of the mysterious behaviour attributed to its conduct. Reflection on the structure of the argument allows us to recognize a sleight of hand in its application to the description of the machine's activity. This involves consideration of a gedankenexperiment which underlies the supposed defiance of Bell's inequality. Section 3 is devoted to the structure of the material problem of quantum physics that the professor would have us ignore in deference to thinking about his wondrous machinery. We shall find that the actual problem under consideration involves a system of restrictive functional restrictions that are ignored in his proposed assessment of its behaviour.

Section 4 presents a Monte-Carlo simulation of the quantum gedankenexperiment which recognizes these functional relations, displaying a frequency of matching lights on the order of 0.375 in situations for which Mermin proclaims his machine to provide only 0.25 . The simulation subscribes completely to the probabilities specified by quantum theory in all appropriate instances. Section 5 then completes the computational analysis of the quantum gedankenexperiment, relying on the application of Bruno de Finetti's fundamental theorem of probability to identify the bounds on the relevant probabilities that quantum theory actually motivates. Quantum theory is quite explicit in refraining from asserting joint probabilities for the outcomes of measurement operators that do not commute. Nonetheless the prescriptions it motivates do imply precise bounds on such probabilities which cohere with the explicit assertions it does provide. My discussion concludes in Section 6 with an overview of what is to be learned from this exercise, and a recognition of precursings of the analysis here already aired in the technical physics literature.

\section{Mermin's Machine and Its Puzzling Properties}

From a box, labeled $c$ in Figure 1, two apparently indistinguishable balls are ejected in opposite directions toward identical receivers at stations labeled $a$ and $b$. There are no discernible connections between these components of the machine, $a, b$, and $c$. Each receiver has a dial on its face that can be positioned to any one of three settings, numbered 1,2 , and 3 . Neither receiver is advised of the dial setting on the other receiver. In whatever way this pair of dials are set, when the balls enter the receivers at the stations, each station will flash one of two lights, coloured Green and Red. The results of a sequence of machine experiments are recorded using ordered notation such as $12 G R$. This would designate that an observation was made with the dial at $a$ set to 1 and that at $b$ set to 2, and that the coloured light observed at $a$ is Green while that at $b$ is Red. Thus, a

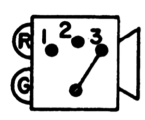

(a)
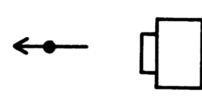

(c)

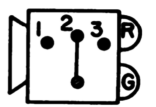

(b)

Figure 1. "The complete device. (a) and (b) are the two detectors. (c) is the box from which the two particles emerge." The original caricature of Mermin's mysterious machine, reprinted with permission from The Journal of Philosophy 78(7): 397-408, 1981. 
sequence of such observations at various dial settings might look something like $12 G G, 31 R G, 13 G R, 22 R R, 32 R R, 12 R G, 21 G R$, and so on.

Here is how the machine works. A pair of apparently identical balls is ejected from box $C$ in opposite directions toward the detectors $A$ and $B$ at whatever dial settings are arranged there, and the signal lights are observed. Once the result is recorded at these settings, another pair of balls is sent out to the detectors in whatever numbered dial settings are then arranged for them. Such experimentation continues sequentially with new pairs of indistinguishable balls. When the dials at $A$ and $B$ happen to be set to the same number for any run, then the colours of the flashing lights are always observed to be either both Red or both Green, with equal frequencies of $1 / 2$ as $G G$ and as $R R$. On the other hand, when the dials are set at different numbers, the signal lights flash the same colour $1 / 4$ of the time, and flash different colours $3 / 4$ of the time. In the former cases, half of the time the identical colours show $G G$, and half the time they show $R R$. In the latter cases, half of the time the flashing colours show $G R$, and half the time they show $R G$. There is no apparent regularity in the orders of their appearance.

The puzzling question and the source of the mystery involved concerns the determination of what could account for such observable results. It seems odd that the signal behaviour at each detector depends on the dial setting at the other detector, yet there is no obvious way for the two receivers to communicate with one another as to the positions of their dial settings. Proposed as a solution is that while the two balls sent to $A$ and $B$ are apparently identical to one another in every way, the character of each pair may be different in successive runs in a way that is not noticeable to the eye. On any given run, the two balls may be somehow encoded each with the same one of eight possible labels: $G G G, G G R$, $G R G, R G G, G R R, R G R, R R G$, or $R R R$. During a long sequence of runs, the source bin of the pairs of balls provides equal numbers of balls encoded with each of these eight configurations, in a random order. When either ball from an identically encoded pair such as $R G R$, for example, enters a detector station, the signal light would flash Red if the dial at that station were set at 1 , would flash Green if the dial were set at 2 , and would flash Red if it were at 3 . That is, whatever the encoded message on the pair of balls may be, the colour flashed at each detector would match its dial setting with the associated colour designated at that position on the ball's encoding string.

Such a scheme would easily account for the fact that when the identically encoded pair of balls enter the two detectors, the signal lights would always flash the same colour if the detector dials were set to the same number. The balls would be coloured either both Green or both Red, depending on the specific identical encoding of the pair of balls and the setting of the dials. But what if the dials at $A$ and $B$ point to different number settings?

\subsection{Mysterious Behaviour: Can You Believe It?}

The professor pronounces that if such a scheme were in vogue, the proportion of runs in which the lights signal the same colour would exceed $1 / 3$ whenever the 
dials are set differently at the two stations. This would obviously defy the known result that the frequency with which matching lights are observed in such situations equals $1 / 4$. Here is his reasoning. The dial settings at $A$ and $B$ are different from one another in six of the nine paired dial settings: $12,13,21,23,31$, and 32. If the encoded message were $R R R$ or $G G G$, the signal lights would always shine the same colour as the encoded ball enters the detectors at these settings. Now consider any encoded message that involves two designations of one colour and one of the other, for examples $R R G$ or $G R G$. In response to such an encoding, say $R R G$, the colour signals at the two stations would be the same for two of the six possible dial settings of the receivers (settings 12 and 21). In the other four settings $(13,23,31$ and 32$)$ the colour signals would be different. Thus, when responding to a long sequence of balls encoded in any of the eight ways, the lights will signal the same colour in at least $1 / 3$ of the runs when the dial settings differ. Responding to two of the codes they always would match, while to any one of the other six codes the coloured lights would match at two of the six differing dial pairings. This argument is said to display the mysterious character of this machine. The encoding of the balls would suffice to explain why the flashing lights show the same colour when the dials are set identically at $A$ and $B$. However, when the dials are set to different numbers, the encoding scheme would seem to imply that at least $1 / 3$ of the observations should exhibit matching colours. Mysteriously, the machine is known to produce matching colours in only $1 / 4$ of the runs with such settings.

An invisible encoding of the balls seems to contradict the facts of the empirical observations of the machine performance. It appears that the proposal of the hidden encoding cannot account for the facts. No other proposal has been offered that can account for the assured matching light signals whenever the dials happen to be set the same. There must be some mysterious connection between the machine components and the observation process itself to account for the facts.

Would you like to dwell on this puzzle yourself for a while if you have not already done so? Literally thousands upon thousands of people have done so, and have been taken in by a sleight of hand in the argument. Without warning or fuss, Professor Mermin has switched the setting of the game on us! Rather than counting the spin products as each pair of balls enters the machine at a dial setting, he is counting the spin products for each pair of balls as it would pass all six of the mixed dial settings. His reported lighting statistics pertains to one game, and his counting of the matching colours pertains to another, two completely different games. As we shall see, it makes sense to consider both games, but they are different for different reasons. We require some more thinking.

\subsection{The Sleight of Hand}

We could use a random number generator both to simulate both the behaviour of the mysterious machine and to simulate the emission of colour-coded balls, with quite different results. In the first case, it is easy enough to generate colour 
signals that always appear randomly as $G G$ or $R R$ when the dials are set identically, but that appear in each of these ways only $1 / 8$ of the time when the dials are set differently, while appearing then as $G R$ and $R G$ each $3 / 8$ of the time. In the second case we would randomly pick an encoding design for the emitted pair of balls and a paired dial setting for the receivers, determining the light signals from the coding rule. These latter results would not match the operations of Mermin's machine. Examine firstly the columns of Table 1. These each display the light signal responses of the machines at one of the nine dial settings to individual pairs of the eight ball encodings listed along the rows, as described in Mermin's proposal. In columns for which the dials are set differently, the light signals are matching for $1 / 2$ of the ball codings, not $1 / 4$. The proportion of matching lights would reduce to $1 / 3$ only if balls coded $G G G$ or $R R R$ were never introduced to the detectors, while the six mixed encodings were introduced at random. The proportion could reduce to $1 / 4$ only if the distribution of emitted ball encodings varied according to the paired dial settings.

Table 1. Encoded messages and their induced responses. The minus symbol (-) designates the display of matching light colour signals, and the plus symbol (+) designates different coloured lights.

\begin{tabular}{cccccccccc}
\hline Dials & 11 & 12 & 13 & 21 & 22 & 23 & 31 & 32 & 33 \\
\hline Setting & 1 & 2 & 3 & 4 & 5 & 6 & 7 & 8 & 9 \\
$G G G$ & - & - & - & - & - & - & - & - & - \\
$G G R$ & - & - & + & - & - & + & + & + & - \\
$G R G$ & - & + & - & + & - & + & - & + & - \\
$R G G$ & - & + & + & + & - & - & + & - & - \\
$G R R$ & - & + & + & + & - & - & + & - & - \\
$R G R$ & - & + & - & + & - & + & - & + & - \\
$R R G$ & - & - & + & - & - & + & + & + & - \\
$R R R$ & - & - & - & - & - & - & - & - & - \\
\hline
\end{tabular}

However, the professor motivated his claims regarding matching-colour frequencies among encoded balls by an argument based on a different situation: experimental results from sending each single pair of encoded balls to detectors at all nine dial setting pairs. His count of two matching lights among six observations arose from observing each pair of mixed-encoded balls such as RRG as it enters all six distinct station pairings with differing dial settings: 12, 13, 21, 23, 31,32 . These counts are exemplified in the rows of Table 1 . To propose that counts from this experiment can represent counts from the original experiment (each pair of balls addresses only a single setting of the dials) amounts to a sleight of hand.

Now who said anything about subjecting a pair of encoded balls to all nine of the paired dial settings? In the operations of the machine which exhibit $1 / 4$ matching lights at different dial settings, each pair of balls is ejected toward a single setting of the dials at stations $A$ and $B$, and the result might be recorded as 
something such as $32 G R$ or $13 R G$. But not both!... not to speak of results of this pair of balls sent to the receivers at the seven other dial pairings. If we would like to study the observed light signals when any single pair of encoded balls is sent to the detectors at all nine paired dial settings, we would require a recording structure more elaborate. We shall have reason to make such a study if we are to examine the relevance of the machine behaviour to the touted violation of Bell's inequality by the probabilities of quantum physics, and we shall.

Suppose we order the detector dial settings as $11,12,13,21,22,23,31,32,33$, and send each pair of identical uncoded balls to all of them. Designating matching-light-colour observations by a -1 and mixed-light-colour observations by +1 , the experimental results would be recorded not merely by something like $13 R G$, but rather something like $(-1,-1,+1,+1,-1,+1,-1,+1,-1)$. Recognizing that the vector components 1,5 , and 9 must all equal -1 because the light colours surely match at these settings, it would appear there would be scope for a sizeable number of distinct observation vectors to arise from such a 9-ply experimental run, perhaps even $2^{6}=64$ of them if the other six might each be either -1 or +1 . We shall learn about this in time when we address the actual gedankenexperiment of quantum physics that the mysterious machine is proposed to emulate.

In order to make a Monte Carlo experiment of the imagined scenario, sending each pair of balls to all nine dial-pair settings at the stations, we would need to observe the light signal responses at every one of them. The accumulating data matrix would have size $N \times 9$ rather than merely $N \times 1$. Of course we shall want to use appropriate quantum probabilities when generating such a sequence, and we shall. (These, remember, involve matching coloured light probabilities of 1/4 when the two dials are set differently.) When we do this in the context of a real quantum experiment, surprisingly we shall find frequencies of matching lights exceeding 1/3 among the different-dial-setting runs, just as Mermin has tendered in his consideration of the encoded balls. The same quantum probabilities that generate his results of $1 / 4$ matching light signals in runs on a sequence of balls each sent to a single dial setting also generate results of matching signal frequencies exceeding 1/3 when each pair of balls addresses all nine settings. There is nothing mysterious about it. What has been missed in Mermin's accounting are the same type of functional relations among the spin-products in a gedankenexperiment that Aspect/Bell missed in their simpler polarization experiment with paired photons. It will take a while to explain the situation.

However there is another peculiarity to be noticed in the presentation of $\mathrm{Ta}$ ble 1. While you follow the professor across a row for any mixed-colour encoded ball in noting the two of six matching light colour results when the two dials are set differently, notice also that there are only four distinct rows of nine-vectors that can possibly result from the scheme using the eight types of ball encodings. There are only eight rows to the Table, and the final four rows duplicate the first four rows, listed in reverse order. Just a few paragraphs ago we were imagining the possibility of several possible nine-vector observational results in the second 
scenario of machine operation, as many as 64 . Now it is evident that the vector $(-1,-1,+1,+1,-1,+1,-1,+1,-1)$ which we suggested as an exemplary possibility would not be a possibility at all under the encoded-ball scheme, despite it identifying matching lights in two of the six paired dial settings that differ. In fact, there are only four distinct nine-vectors of result possibilities arising from the eight encoding designs. The bottom line for now is that this scheme of sending encoded balls to detectors at all nine dial settings is a proposition completely different from that which yields the proclaimed results of Mermin's machine. We shall sort this all out forthwith.

To clarify the situation requires a diversion into the real quantum experiment that the professor would have us ignore while we are enticed to marvel at his mysterious machine. My plan is to begin with a presentation of the relevant practical quantum experiment that can be and has been conducted many times. Then we shall embellish the context to a gedankenexperiment designed to assess the implications of Einstein's principle of local realism and his challenge to the completeness of quantum theory. This is the context in which the specification of Bell's inequality is entertained, and the context for which Mermin's second version of the game is appropriate as an emulation. It is only once we recognise the structure of this matter that we will be able to identify prospective quantum probabilities when a single pair of balls visits all nine designs of dial settings.

Once we have studied the structure of the real quantum experiment and its associated gedankenexperiment, we shall design and conduct a Monte Carlo experiment as a prelude to a complete analysis of the entire situation based strictly on the limited claims of quantum theory. Surprisingly, the simulation also exhibits matching light frequencies exceeding $1 / 3$ under conditions that Mermin proposes as mysterious. But the Monte Carlo simulation will not constitute the end of our analysis. As with my solution to the simpler experimental context of Aspect/Bell in [3], we will find in the complete analysis that quantum theory does not propose a joint probability distribution over the complete space of possible gedanken observations. Rather, in its current incomplete form it specifies a multi-dimensional polytope of such distributions, and explicitly renounces any prospect for refining it. The simulation design, while natural, is not the only design that QM theory would allow, and we shall see why. This is all sounding fairly complicated. However, it is merely a matter of plodding on to sort things out.

\section{What Are We Really Talking about?}

Professor Mermin understands the mystery to convey that while there are no obvious physical connections between the three pieces of the experimental device, $A, B$, and $C$, the attempt to explain its experimental features by unobservable instructions encoded within the balls is futile. Such an explanation might account for the specified observable outcomes of the machine when the dials are at $A$ and $B$ set identically as 11,22 , or 33 , but it seems to provoke specific observations of flashing lights that do not match what we experience when running the 
machine at other dial pairings. The alternative he proposes is to recognise that indeed the operation of the recorders actually is connected in some mysterious way, suggesting "connections of no known description, that serve no purpose other than relieving us of the task of accounting for the behavior of the device in their absence." This is the purportedly mysterious behaviour of quantum mechanics as is currently widely promoted. However Mermin engages such speculations no further, as the task proposed for his exposition was merely to state the conundrum, not to resolve it. The parable is concluded.

After completing his description of the mystery, the professor presents an insightful discussion of the relevance of the parable to issues raised by Einstein, Podolsky, and Rosen [5] in their proposition that the theory of quantum mechanics must be incomplete. While they had presented arguments that may appear telling regarding the activity of the machinery when the dials are set identically at $A$ and $B$, their arguments appear to fail in situations in which the dials are set differently. This was a situation they did not assess, consumed as they were in their article with claims about the reality of quantum states and their observations that could be predicted with certainty. The implications for quantum behavior portrayed in the parable by different dial settings at $A$ and $B$ did not become evident until the startling research results of John Bell. These have been understood to display that if one presumes Einstein's principle of local realism and the relevance of hidden variables, then the specifications of quantum theory defy some standard inequalities of probability theory.

Mermin's exposition concludes with a description of the contextual quantum experiment that the parable is meant to portray, emphasizing that such detail can be conveniently ignored while the significance of the mystery is absorbed in awe. This is a well-known quantum experiment involving a pair of electrons that are propelled in opposite directions toward identical detecting devices of Stern-Gerlach magnets at stations $A$ and $B$, each of the magnets oriented at one of three specific angles within the plane perpendicular to the incoming electrons. The detectors identify the magnetic spins of the electron pair, each in either the direction "up" or "down", denoted by $A=+1$ or $A=-1$, and similarly for the value of $B$. Rather than ignoring the experimental physics as suggested, the remainder of my exposition now is oriented to a detailed assessment of the exact specification of this experiment and the proclamations of quantum theory that concern it. We shall find that the parable fails to represent the situation adequately, for the same reason that Aspect's assessment of Bell's inequality fails in the simpler case of a pair of photons presented to two paired polarization angles. It is a mathematical error of neglect.

\subsection{The Quantum Experiment in Its Gedanken Extension}

Remember that the simple quantum gedankenexperiment of Aspect/Bell concerned a cerebral assessment of possibilities for the combined result of four practical experiments, each of which can be engaged, but for which the engagement of all four simultaneously is recognized as impossible. The same style of 
investigation will pertain to our considerations now. We shall examine the (im)possible imagined results of a nine-ply Stern-Gerlach thought experiment conducted on a single pair of electrons. This is the context to which Bell's inequality pertains and in which the principle of local realism is relevant. Now it is a pair of electrons that are propelled in opposite directions toward the stations of Alice and Bob. In a real experiment, each is charged with observing one of the pair as it passes a magnet oriented in one of three different directions relative to vertical up and down. The vertical position is designated as the zero position, and the other two are directed in twists of negative and positive angles relative to this zero. The possible pairings of these magnet orientations at the two stations specify $3 \times 3$ possibilities for a paired choice of them at the two stations during any experimental run. The vector outcome of spin-products occurring in a run of a thought experiment, sending a single electron pair to all nine paired magnet orientations, will be denoted $G_{9}$, the " $G$ " standing for "gedankenvector".

Initially we shall designate the three possible magnet orientations of each spin observation variable by the subscripts $n, Z$, or $p$, so to represent its alignment relative to vertical as negative, zero, or positive. We may write $A_{p}$ or $B_{z}$, for examples. When referring to a spin observation at a generic magnet orientation we may write the quantities $A$ and $B$ without subscript, or we may use a subscript letter " $d$ " considered as a variable. Eventually we shall assess the specific setup in which the two chosen magnet orientations differ by the angles $-120^{\circ}, 0^{\circ}$, and $+120^{\circ}$. This is the setup relevant to the probability assessments prescribed in Mermin's parable. Deliberations of quantum theory specify probabilities for the possible paired observations of spins as up or down at the two stations, casually denoted as $P_{++}, P_{+-}, P_{-+}$, and $P_{--}$appropriate to any such angle pairing. Equivalently, they specify the expectation of the spin product, $E(A B)$.

In the gedankenexperiment, Alice and Bob will observe the spins of a pair of electrons in every paired directional setting of their magnets. Their respective observations named $A$ and $B$ would be recorded as either +1 or -1 to designate an observation of spin "up" or "down". We shall denote the possible results of their nine paired observations by product event designations such as

$\left(A_{n}=+1\right)\left(B_{z}=-1\right), \quad\left(A_{n}=+1\right)\left(B_{z}=+1\right), \quad\left(A_{p}=+1\right)\left(B_{n}=+1\right)$, or $\left(A_{z}=+1\right)\left(B_{z}=-1\right)$, and so on, results exhibiting spin-products of $-1,+1,+1$ and -1 respectively. My use of arithmetic notation means that each of these product events indicates whether the joint observation of spin values at site $\mathrm{A}$ and site $\mathrm{B}$ arises in a particular configuration or not. There will be nine of them. The number of prospective nine-tuples of observation products could be as large as $2^{9}=512$. This number of possibilities will be reduced shortly on account of theoretical speculation and on account of the particular directional angles we employ in the experiment's design.

We shall begin by considering a list of all the possible results of the paired observations at $A$ and $B$ that could be entertained according to Einstein's contentious (and currently widely rejected) "locality" condition. This involves a proposition that lies outside the technical domain of quantum theory. It accepts that 
the electron spin observation made by Alice in any specific magnet orientation is a result assessed with a quantum probability entangled with that of Bob's magnet direction in that instance. However it proclaims that no matter what her magnet orientation may be in any particular experimental run, Alice's spin observation in this instance would be the same (either up or down) no matter what be the corresponding orientation of Bob's magnet and his spin observations in imagined companion experiments on the same pair of electrons.

The reason such a claim lies outside the scope of quantum theory is that the distinct operator matrices for observation of a single pair of electrons addressing two different designs of magnet orientations do not commute. Thus, quantum theory itself says nothing about their joint product results at the two designs. The complete experiment, a thought experiment, presumes that a single pair of electrons passes by the two magnets in all nine of their paired orientations. The principle of local realism stipulates that if Alice's spin observation is, say, +1 in a specific magnet orientation when Bob's relative orientation angle is $+120^{\circ}$, then Alice's would also equal +1 in this instance if Bob's magnet were oriented relatively at $0^{\circ}$ and/or at $-120^{\circ}$ as well. Bob's spin observations might equal either +1 or -1 in either case. Although Alice's actual measurement for a particular electron spin is proposed to be invariant with respect to the setting of Bob's detection angle, this principle respects nonetheless an assertion of entanglement of the electrons. This understanding derives from the specification of quantum theory that $P[(A=+1)(B=-1) \mid \theta]=\frac{1}{2} \cos ^{2}(\theta / 2)$ at any single relative angle setting, where $\theta$ is the relative angle between Alice's and Bob's magnet orientations. Equivalently the specification is $E(A B \mid \theta)=1-2 \cos ^{2}(\theta / 2)$. This is the relevant prescription of quantum theory.

The principle of local realism implies that in measuring the spins at all nine angle orientation pairs for the gedankenexperiment, each of the observers would register only three distinct spin values. According to this premise, each of the observed values of $A_{n}, A_{z}$, and $A_{p}$ in the nine-ply experiment would be the same no matter which of the station $B$ magnet orientations it were paired with, $B_{n}, B_{z}$, or $B_{p}$. The same would hold for the observation values of the $B$ s. These six observation values would display themselves among nine specific observation pairs of the form $\left(A_{d}, B_{d}\right)$. There appear to be only $2^{6}=64$ conceivable instantiations of these six spin observations that would respect the principle of local realism in any run of the gedankenexperiment. However, the probabilistic assertions of quantum theory reduce the number of these possibilities still further. Consider the prescription of quantum theory pertinent to a any experimental setup in which Alice's and Bob's magnet orientations are identical, and we measure the spin values $\left(A_{n}, B_{n}\right)$, or $\left(A_{z}, B_{z}\right)$, or $\left(A_{p}, B_{p}\right)$. Quantum theory stipulates that in any such experiment we must observe opposite spin values at stations $A$ and $B$. For the quantum prognostication stipulates that $P\left(A_{d} B_{d}=-1 \mid \theta=0\right)=1$, and equivalently $E\left[A_{d} B_{d} \mid(\theta=0)\right]=-1$ whenever the two magnet orientations are identical. It is impossible for the spin observa- 
tions at Alice's and Bob's stations to be the same when their magnet orientations are the same. An implication of these quantum probabilities along with the principle of local realism is that there are not 64 possible results of the gedankenexperiment, but rather only 8 . Let's examine them.

\subsection{Specifying the Possible Results of the Stern-Gerlach Gedankenexperiment}

Let's cut to the quick, and present for comment a list of all possible results of the $3 \times 3$ gedankenexperiment that might be observed for a single pair of electrons passing the magnets in all of their possible relative orientations. The possibilities constituting the experimental ensemble are presumed to be limited by the principle of local realism and the assertions of quantum theory, particularly as they are relevant to component experiments in which the orientations of Alice's and Bob's magnets are identical. That is, we shall examine the "realm matrix" of all possibilities for the unknown observation vector $\boldsymbol{G}_{6} \equiv\left(A_{n}, A_{z}, A_{p}, B_{n}, B_{z}, B_{p}\right)^{\mathrm{T}}$ that could result from the running of such a nine-ply-thought-experiment. These are followed by the vector of spin-products

$$
\boldsymbol{G}_{9}=\left(A_{n} B_{n}, A_{n} B_{z}, A_{n} B_{p}, A_{z} B_{n}, A_{z} B_{z}, A_{z} B_{p}, A_{p} B_{n}, A_{p} B_{z}, A_{p} B_{p}\right)^{\mathrm{T}}
$$

that such an array of spin observations would imply for the nine paired magnet orientations. Partitioned (for reasons to be seen) vertically into three sections and horizontally into two for examination, the realm matrix appears as

$$
\left(\begin{array}{c}
A_{n} \\
A_{z} \\
A_{p} \\
B_{n} \\
B_{z} \\
B_{p} \\
* * * \\
A_{n} B_{n} \\
A_{n} B_{z} \\
A_{n} B_{p} \\
A_{z} B_{n} \\
A_{z} B_{z} \\
A_{z} B_{p} \\
A_{p} B_{n} \\
A_{p} B_{z} \\
A_{p} B_{p} \\
* * * \\
{ }^{2} A_{n} B_{n} \\
2 \\
A_{n} B_{z} \\
3 \\
A_{n} B_{p} \\
{ }^{6} A_{z} B_{p}
\end{array}\right)=\left(\begin{array}{rrrrrrrrr}
1 & 1 & 1 & 1 & * & -1 & -1 & -1 & -1 \\
1 & 1 & -1 & -1 & * & 1 & 1 & -1 & -1 \\
1 & -1 & 1 & -1 & * & 1 & -1 & 1 & -1 \\
-1 & -1 & -1 & -1 & * & 1 & 1 & 1 & 1 \\
-1 & -1 & 1 & 1 & * & -1 & -1 & 1 & 1 \\
-1 & 1 & -1 & 1 & * & -1 & 1 & -1 & 1 \\
* & * & * & * & * & * & * & * & * \\
-1 & -1 & -1 & -1 & * & -1 & -1 & -1 & -1 \\
-1 & -1 & 1 & 1 & * & 1 & 1 & -1 & -1 \\
-1 & 1 & -1 & 1 & * & 1 & -1 & 1 & -1 \\
-1 & -1 & 1 & 1 & * & 1 & 1 & -1 & -1 \\
-1 & -1 & -1 & -1 & * & -1 & -1 & -1 & -1 \\
-1 & 1 & 1 & -1 & * & -1 & 1 & 1 & -1 \\
-1 & 1 & -1 & 1 & * & 1 & -1 & 1 & -1 \\
-1 & 1 & 1 & -1 & * & -1 & 1 & 1 & -1 \\
-1 & -1 & -1 & -1 & * & -1 & -1 & -1 & -1 \\
* & * & * & * & * & * & * & * & * \\
-1 & -1 & -1 & -1 & * & -1 & -1 & -1 & -1 \\
-1 & -1 & 1 & 1 & * & 1 & 1 & -1 & -1 \\
-1 & 1 & -1 & 1 & * & 1 & -1 & 1 & -1 \\
-1 & 1 & 1 & -1 & * & -1 & 1 & 1 & -1
\end{array}\right)
$$

Within the first vertical partition of the named vector appear the six observa- 
tions of Alice and Bob in the performance of the nine experiments: $\left(A_{n}, A_{z}, A_{p}, B_{n}, B_{z}, B_{p}\right)^{\mathrm{T}}$. While each component of the $6 \times 1$ vector can equal either -1 or +1 , the components of the second triple in any column, $B_{n}, B_{z}$, and $B_{p}$, must be the negative values of the first three components of that column. This is a prescription of quantum theory. The spin directions of Alice and Bob must oppose one another when their magnet orientations are the same. Each of the eight columns displaying an array of Alice's spin possibilities at her three magnet orientations is accompanied by a display of Bob's opposing spin values at his matching orientations. To the contrary, when the magnet orientations of Alice and Bob differ, then a spin observation at $A$ in either direction might be accompanied by a spin observation at $B$ in either direction as well. The spin-product might equal -1 or +1 . Composing the columns of the middle partitioned section of this realm matrix are the arithmetical products of each of the three $A$ 's with each of the three $B$ 's appearing in the same column. There are nine such product quantities. The product $A_{d} B_{d}$ equals -1 whenever the magnet directions at stations $A$ and $B$ are identical. The third partitioned sections of the named quantity vector and of its realm matrix merely repeat rows $1,2,3$, and 6 of the middle partition. We shall discuss them when it becomes appropriate.

\subsection{A Substantive Recognition: Functional Relations among Spin-Products}

A substantive matter to recognise about this realm matrix is that the four columns of the right partition of the middle section constitute a folded replica of the columns of the left partition of that section. The middle section of column 5 is identical to that of column 4. That of 6 is identical to column 3, and so on, until the midsection of column 8 is identical to that of column 1 . Thus, the middle partition of the realm matrix has only 4 distinct columns, rather than 8 as the top partition appearing above it does. Moreover, the middle partition matrix has only four distinct rows rather than nine as one might naively suspect. This fact has important ramifications for the analysis of the hypothetical problem of nine distinct Stern-Gerlach experiments on the same pair of electrons!

In the first place, the realm matrix for the spin-product vector resulting from the gedankenexperiment on a single pair of electrons consists of only the left half of the middle partition matrix above, a $9 \times 4$ matrix. For reference in ensuing discussions, we shall refer to this realm matrix for the vector of nine spin-product observations in the gedankenexperiment as $\boldsymbol{R}_{9,4}$. What is more, some of the rows of this complete matrix are obtainable via specific functions of other rows in that section. As long as any two rows of $\boldsymbol{R}_{9,4}$ constitute the cartesian product $\{-1,+1\}^{2}$, the remaining seven rows are determined by a function of them, an "into" mapping with the structure $\{-1,+1\}^{2} \rightarrow\{-1,+1\}^{7}$. Consider, for example, the rows 2 and 3 of $\boldsymbol{R}_{9,4}$. Their column pairs exhaust the cartesian product $\{-1,+1\}^{2}$, constituting the domain of a function. For each of these pairs in the domain, the remaining seven columns provide a unique vector within $\{-1,+1\}^{7}$. We shall designate this function with the notation $23 \rightarrow 1456789$. 
In fact there are twelve such functional relations that inhere within the structure of the realm matrix $\boldsymbol{R}_{9,4}$. Using the same functional notation, we can list them as:

$$
\begin{array}{lll}
23 \rightarrow 1456789 & 34 \rightarrow 1256789 & 47 \rightarrow 1235689 \\
26 \rightarrow 1345789 & 36 \rightarrow 1245789 & 48 \rightarrow 1235679 \\
27 \rightarrow 1345689 & 38 \rightarrow 1245679 & 67 \rightarrow 1234589 \\
28 \rightarrow 1345679 & 46 \rightarrow 1235789 & 78 \rightarrow 1234569
\end{array}
$$

The first of these arrows denotes the functional relation among the columns of spin-products we have just described. It denotes a mapping from $\{-1,+1\}^{2}$ into $\{-1,+1\}^{7}$. The subsequent arrow structures all describe functions as well. All that is required is that the two rows of $\boldsymbol{R}_{9,4}$ corresponding to the domain variables exhibit among their column pairs the component vectors of $\{-1,+1\}^{2}$. This list of functional relations embedded in the realm matrix is exhaustive. There are six paired spin products corresponding to experiments with different magnet orientations at the stations of Alice and Bob, these being the components $2,3,4,6,7$, and 8 of any spin-product vector. There are ${ }^{6} C_{2}=15$ possible choices of two spin-products to consider as elements of a possible function domain. However three such choices of two of them fail to provide a valid function domain: $(2,4),(3,7)$, and $(6,8)$. That is, attempted mappings of 24 onto 1356789 , of 37 onto 245689 , and of 68 onto 1234579 all fail to identify a function. For example, the column pairs from rows 2 and 4 do not exhaust $\{-1,+1\}^{2}$. Furthermore, when they repeat they correspond with two distinct tentative objects in $\{-1,+1\}^{7}$. The relation this structure provides for consideration does not constitute a function.

In the second place worthy of note, the twelve embedded functional relations among the spin products we have recognized are not linear. If they were, the rank of the realm matrix $\boldsymbol{R}_{9,4}$ would be only two, but it is four! This is a feature crucial to the implications of quantum theory for assessing the prospective results of the gedankenexperiment. Quantum theory makes specific expectation (and probability) assertions regarding the spin product possibilities for any two domain variables among our listed functions, considered as distinct isolated experiments. If the functional relations we have enumerated were linear then these would imply precise expectations for the range variables. As it is, the assertions of quantum theory will stipulate only bounds on the expectations for the spin-products of the range variables in any such case. This situation inheres some intrigue. The conditional distribution for any seven products in a range vector given the results of the domain vector would be degenerate at their function value. Yet their joint probability distribution with the domain variables cannot be determined precisely. We shall see more of this.

A further remark of note concerns the paired repetitions found among rows 2 and 4, rows 3 and 7, and rows 6 and 8 in the central vertical partition of this realm matrix. These identities exhibit specific symmetries among components of 
the spin-product vector of a gedankenexperiment run: the commutativity of spin-product observations with respect to the orientations of their detecting magnets. Noting the names of the spin-product quantities whose observation possibilities constitute the repeating rows, these repetitions specify that $A_{n} B_{z}=A_{z} B_{n}, A_{n} B_{p}=A_{p} B_{n}$, and $A_{z} B_{p}=A_{p} B_{z}$ in any imagined run of the experiment. This feature of symmetry will come to bear on our computations of probability bounds for gedankenresults deriving from the claims of quantum theory, restricted by its avowed uncertainty principle.

A final surprise can be seen among the columns of the partition matrix $\boldsymbol{R}_{9,4}$. Although we have mentioned nothing at all about encoded balls while constructing it, it is apparent that the columns of $\boldsymbol{R}_{9,4}$ match the row designations in Table 1 which repeat themselves. These exhibit the light signal responses of Mermin's mysterious machine to his explanatory suggestion of encoded balls. We shall come back to this recognition too in due time.

\section{A Simulation Experiment, Using QM Motivated Probabilities}

We shall now capitalize on our recognition of the functional relations embedded in the realm matrix $\boldsymbol{R}_{9,4}$, by conducting a simulation experiment appropriate to the restricted experiment. It represents the situation Professor Mermin thought he was assessing when he evaluated the behaviour of his mysterious machine in response to colour-encoded balls at all nine dial-pair combinations. Our simulation is meant to elucidate the structure that the theory of quantum mechanics designates as appropriate to the assessment of Bell's inequality in the context of its associated gedankenexperiment. It will generate a sequence of twelve million gedankenvectors $\boldsymbol{G}_{9}$, these being the simulated spin-products of electron pairs that each pass all nine paired magnet orientations, obeying the probabilistic prescriptions of quantum theory.

The experiment will be conducted in twelve configurations, corresponding to the twelve functional relations enumerated in Section 3.3. Data are generated that correspond to a gedankenexperiment of Stern-Gerlach apparatus with the angled magnet direction possibilities for both Alice and Bob set at all three of their orientations relative to vertical in the $(x, y)$ plane, $-120^{\circ}, 0^{\circ}$, and $+120^{\circ}$. This plane of directions is perpendicular to the direction of the incident electrons, exactly as described in the conclusion to the "Quantum Mysteries for Anyone". We shall denote the relative angle between Alice's and Bob's magnet orientations in any paired direction setup by $\theta=d_{B}-d_{A}$. The nine paired direction possibilities yield orientations that differ by angles of $\theta=0^{\circ}$ when the $\mathrm{A}$ and $\mathrm{B}$ observations are $\left(A_{n}, B_{n}\right),\left(A_{z}, B_{z}\right)$, and $\left(A_{p}, B_{p}\right)$; of $\theta=+120^{\circ}=-240^{\circ}$ when the $\mathrm{A}$ and $\mathrm{B}$ observations are $\left(A_{n}, B_{z}\right),\left(A_{z}, B_{p}\right)$, and $\left(A_{p}, B_{n}\right)$; and of $\theta=-120^{\circ}=+240^{\circ}$ when the $\mathrm{A}$ and $\mathrm{B}$ observations are $\left(A_{n}, B_{p}\right),\left(A_{z}, B_{n}\right)$, and $\left(A_{p}, B_{z}\right)$. Rows 1,5 , and 9 of our realm matrix $\boldsymbol{R}_{9,4}$ correspond to an orientation difference angle $\theta=0^{\circ}$; row numbers 4,8 , and 3 pertain to the difference angle $\theta=-120^{\circ}$; and rows 7,2 , and 6 pertain to the difference angle $\theta=+120^{\circ}$. 
The simulation begins with a routine pertinent to the spin-product function $23 \rightarrow 1456789$. It first generates observation values for components 2 and 3 of the spin-product vector independently according to standard QM specifications of probabilities for differing spin observations at these magnet orientation pairings. These prescribe the quantum probabilities

$$
P\left[A_{n} B_{z}=-1 \mid \theta=-120^{\circ}\right]=1 / 4=P\left[A_{n} B_{p}=-1 \mid \theta=+120^{\circ}\right],
$$

which correspond to the frequencies reported by Mermin in the observations of his machine. The coloured lights match only $1 / 4$ of the time when the stations' magnet orientations differ.

Appropriately then, from each eventuality of the two outcome values so generated for components 2 and 3, the associated spin-product values for components $1,4,5,6,7,8,9$ are computed according to the functional rule we have designated with the notation $23 \rightarrow 1456789$. In each of these cases, the distribution of these latter component values given the spin-product pair simulated for the components of the domain is degenerate on their function value. This derives from the structural features of the possible spin-product observations. From one million such generations the number of occurrences of -1 are counted and recorded in the spin-product columns 1 through 9.

The results of the simulation yield surprising counts which can be displayed as

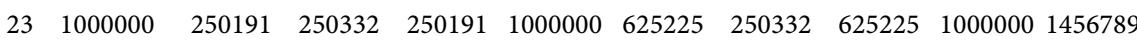

The number at the far left edge of this row of such counts designates the row numbers of function domain observations that were selected by the pseudo random numbers of MATLAB, while the number at the far right edge identify the corresponding rows in the range that were computed via appropriate function rules. Understanding this, you should read this report line as identifying 1000000 counts of spin-product observations -1 in experiment columns 1,5 , and 9. (Mermin's lights always flash the same colour when the dials at $A$ and $B$ read the same.) Counts of -1 amounted to 250191, 250332, 250191, and 250332 as recorded in experiments 2, 3, 4, and 7, respectively, two pairs of which involve repetitions. Finally, identical counts of 625225 were recorded in both columns 6 and 8. (The identical counts in three of the column pairs result from the commuting symmetries which we found to insist that $A_{n} B_{z}=A_{z} B_{n}$, that $A_{z} B_{p}=A_{p} B_{z}$, and that $A_{p} B_{n}=A_{n} B_{p}$ when the experiments run gedankenly in tandem. This feature arises naturally in the function-based generations of the simulation.)

Notice particularly, that the repeated count of 625225 corresponding to spin products $A_{z} B_{p}$ and $A_{p} B_{z}$ differs markedly from the claims of Professor Mermin that the experiment should yield a number near to 250000 in every spin-product column for which the difference in orientation angle is $\theta=-120^{\circ}$ or $\theta=+120^{\circ}$. We can now understand why this has occurred! On account of the functional relations required among the spin-product observations, only a select two of the spin-product values can be generated freely according to QM 
probability relations as specified by the well-known cosine squared equations.

$$
P[(A=+1)(B=-1) \mid \theta]=\frac{1}{2} \cos ^{2}(\theta / 2)
$$

The remaining seven must be determined from the values of these two according to the functional relation we are considering, which binds them all.

Now there is nothing special about the experimental components 2 and 3 which we allowed to be chosen freely by their quantum probabilities. We have seen that there are twelve such domain choices of experimental pairs that can be used to generate the gedanken spin vector. We shall now examine a full array of simulation results deriving the other eleven choices of the function domain as well. They will be found to allay misdirected concerns aired in the parable regarding proportions of matching light colours proclaimed to differ from 1/4. Such concerns are appropriate in real physical experiments in which sequences of electron pairs engage any single pair of differing magnet orientations. But they are not appropriate to a gedankenexperiment motivated by quantum theory and local realism in which each pair passes all nine magnet orientation pairings. Let's look at the complete results.

\subsection{An Array of Simulation Results Generated by Twelve Functional Relations}

Displayed in Table 2 are results of twelve simulation runs that are structurally identical in their generation to those I have already described for the function $23 \rightarrow 1456789$. Distinct runs were based on QM probability assertions applied to the two domain variables of each of the twelve functional relations we have recognized. In each set of runs, spin-products were generated for two appropriate function domain variables using QM probability simulations. Then the remaining seven were computed from these using the relevant function specifications we have identified. Nonetheless the output of each 9-vector generated respects the injunctions of all twelve function rules. Without further ado, Table 2 below presents the results of these simulations, presented according to the same reporting structure used in the previous Subsection. In fact, the first row of this Table repeats the results that were reported there.

Each row of these Simulation Count results is based upon quantum probabilities relevant to a distinct function whose domain rows are displayed in its left edge column. Columns numbered 1,5, and 9 (not counting the edge columns) show that the generated spin-product is negative at all three configurations in which magnet orientations at the sites $A$ and $B$ would be identical. The products of the simulated spin observations yield counts of 1000000 in these columns, exactly as expected in quantum theory. The negative spin-product counts at the other gedanken configurations vary. For example, row 3 of the Table, which identifies in its left edge column the relevant functional relation as $27 \rightarrow 1345689$, exhibits counts of 250096 and 250274 in matrix columns 2 and 7, as expected according the probability specifications of quantum theory applied to a single 
Table 2. The letter D at top left stands for "Domain" of a function. Twelve simulation counts of negative spin-products.

\begin{tabular}{|c|c|c|c|c|c|c|c|c|c|c|}
\hline$D$ & ${ }^{1} A_{n} B_{n}$ & ${ }^{2} A_{n} B_{z}$ & ${ }^{3} A_{n} B_{p}$ & ${ }^{4} A_{z} B_{n}$ & ${ }^{5} A_{z} B_{z}$ & ${ }^{6} A_{z} B_{p}$ & ${ }^{7} A_{p} B_{n}$ & ${ }^{8} A_{p} B_{z}$ & ${ }^{9} A_{p} B_{p}$ & Range \\
\hline 23 & 1000000 & 250191 & 250332 & 250191 & 1000000 & 625225 & 250332 & 625225 & 1000000 & 1456789 \\
\hline 26 & 1000000 & 249641 & 625501 & 249641 & 1000000 & 249912 & 625501 & 249912 & 1000000 & 1345789 \\
\hline 27 & 1000000 & 250096 & 250274 & 250096 & 1000000 & 624192 & 250274 & 624192 & 1000000 & 1345689 \\
\hline 28 & 1000000 & 250188 & 625260 & 250188 & 1000000 & 250060 & 625260 & 250060 & 1000000 & 1345679 \\
\hline 34 & 1000000 & 250777 & 250397 & 250777 & 1000000 & 624338 & 250397 & 624338 & 1000000 & 1256789 \\
\hline 36 & 1000000 & 625459 & 249849 & 625459 & 1000000 & 249814 & 249849 & 249814 & 1000000 & 1245789 \\
\hline 38 & 1000000 & 624890 & 250619 & 624890 & 1000000 & 250277 & 250619 & 250277 & 1000000 & 1245679 \\
\hline 46 & 1000000 & 250093 & 624872 & 250093 & 1000000 & 249855 & 624872 & 249855 & 1000000 & 1235789 \\
\hline 47 & 1000000 & 249640 & 249716 & 249640 & 1000000 & 625256 & 249716 & 625256 & 1000000 & 1235689 \\
\hline 48 & 1000000 & 249483 & 625658 & 249483 & 1000000 & 249411 & 625658 & 249411 & 1000000 & 1235679 \\
\hline 67 & 1000000 & 625506 & 249710 & 625506 & 1000000 & 249974 & 249710 & 249974 & 1000000 & 1234589 \\
\hline 78 & 1000000 & 625491 & 249736 & 625491 & 1000000 & 249681 & 249736 & 249681 & 1000000 & 1234569 \\
\hline \multicolumn{11}{|c|}{ Sum Simulation Counts by Product Column: } \\
\hline & 12000000 & 4501455 & 4501924 & 4501455 & 12000000 & 4497995 & 4501924 & 4497995 & 12000000 & \\
\hline \multicolumn{11}{|c|}{ Proportions of Negative Spin-Products by Product Column: } \\
\hline & 1.0000 & 0.3751 & 0.3752 & 0.3751 & 1.0000 & 0.3748 & 0.3752 & 0.3748 & 1.0000 & \\
\hline
\end{tabular}

experimental magnet settings. However, in columns 6 and 8 of row 3, among the range variables of the constraining function both counts are found to be 624192, not near to 250000 at all!

Similar structures govern the simulation counts in all rows of the count matrix shown in Table 2: two of the column elements of each row exhibit identical counts in the vicinity of 625000 while four column elements are in the vicinity of 250000 , arising as two identical count pairs. No matter which pair of function domains is used to generate the nine columns of results, the counts are always identical in columns 2 and 4, in columns 6 and 8, and in columns 3 and 7. The requirements of commuting spin observations such as $A_{n} B_{z}=A_{z} B_{n}$ are satisfied simply by recognition of the functional relations among spin products.

Summing the twelve columns of these simulation counts (which each arise from 1 million simulated Stern-Gerlach experiments) yields further results of interest. Quite striking in fact are the implied proportions of differing spin-products exhibited at the several paired angle orientations. These proportions are not displayed as three 1's and six 0.25 's as proclaimed by Professor Mermin. The three 1 's surely appear in the expected places, but of the remaining six columns we find all the proportions near to 0.375 , defying his claim to the proportion arising as $1 / 4$. Indeed, the proportions we have generated in the quantum gedankensimulation are reminiscent of the frequency behaviour of encoded balls which had worried him in his parable, motivating him (with many others) to decry the sensibility of Einstein's suggestion of hidden variables in quantum behaviour. Nevertheless, such ball coding was not employed at all in the simulated generation of these results. 


\subsection{Comments, a Qualification, and a Query}

Don't get me wrong. If you would do a sequence of simulation experiments at a specific pairing of differing magnet orientations using quantum probabilities, you would find the spin-product values to equal -1 in close to $1 / 4$ of these cases. However, if you do a long sequence of simulated experiments that gedankenly subjects the electrons to all nine paired magnet angle directions in the way local realism restricts them, you would find the proportion of spin-products equal to -1 at about 0.375 whenever the relative angle between the magnets equals $-120^{\circ}$ or $+120^{\circ}$. This happenstance governs the counts displayed in columns $2,3,4,6$, 7 , and 8 . The result has nothing to do with Mermin's proposed explanation of "the mystery" involving colour-encoded balls. It derives from a recognition of the functional relations embedded into spin-product possibility vectors in the gedankenexperiment. A situation clearly distinct, when the rows are produced by gedankenly submitting each pair of electrons to all nine of the relative angle settings, many elements of the cartesian product $\{-1,+1\}^{6}$ for the unequal magnet angle designs would constitute impossible outcomes of the spin-product functions that govern the experiment. Each of the allowable result vectors respects twelve functional mappings of $\{-1,+1\}^{2}$ onto $\{-1,+1\}^{7}$.

Professor Mermin's fabulous machinery produces no mysterious results at all. The character of the matrix of results would be different, depending on which of the two different ways that the results are generated. This is not surprising.

One way to simulate the gedankenexperiment as supported by the probabilities of quantum theory would be to pick sequentially (randomly, uniformly) one of the functional relations that bind the spin-products $\{-1,+1\}^{2} \rightarrow\{-1,+1\}^{7}$, to generate a vector of nine-tuple observations as we have described. Then pick a functional relation again to generate the next 9-vector of results. Continuing with this process we would generate a sequence of such 9-vectors, and accumulate the counts of negative spin-products at the nine angle pairings across the sequential generations. This process would result in proportions of negative spin-products as appear in the final line of Table 2. However, this result could hardly be claimed to be a definitive prognostication of quantum theory relevant to the gedankenexperiment. Be aware that the nine component vector results of the experimental runs involve simultaneous results of observations at angle pairings whose operator matrices do not commute! Quantum theory explicitly says nothing specific about such impossible experimental results.

So what does quantum theory say, and how can we present it in a complete and concise way? Consider again a single functional relation such as $23 \rightarrow 1456789$. Well, quantum theory is quite specific in identifying a probabilistic structure governing the possible results at either of the two relative angles between the Stern-Gerlach magnets that appear in the function domain. However, it is also quite explicit in denying any motivation for making claims about simultaneous spin-product results at any two relative angle settings for which the observation matrix operators do not commute, these two in particular. The results of this simulation activity I have just proposed could be admissible according to the 
logic of quantum theory, but there is no reasoning that would make the joint distribution they imply definitive. There is no requirement that function domains be picked randomly and uniformly at all, as I have done here. We could generate other distributions of results if we picked them according to some other random scheme.

The way to characterize the complete space of joint probability distributions over gedanken results that cohere with the positive claims of quantum theory is to assess a battery of linear programming computations. These can identify the bounds on probabilities for the range settings of the constraining functions that would cohere with the specifications that quantum theory does provide for results of function domain settings. To produce such an assessment is the burden of our next Section. This involves investigating the implications of Bruno de Finetti's "fundamental theorem of probability" for the nine proposed Stern-Gerlach gedankenexperiments, all performed on the same pair of electrons. We turn to this investigation now.

\section{Characterizing a QM Probability Polytope for Stern-Gerlach Gedankenexperiments via de Finetti's FTP}

If you are not familiar with Bruno de Finetti's fundamental theorem of probability [6], Chapter 3.10, then you should read a brief commentary and description of the theorem in linear programming form which I attach to this essay as an Appendix. In a word, the theorem identifies a linear programming problem that characterizes the bounds on the probability for any event that are required by its coherency with other probabilities that are taken as given. An application of this theorem to the assessment of the Aspect/Bell error was made in Section 7 of my article [3] about supposed inequality violations entitled, "Quantum violations of Bell's inequality: a misunderstanding based on a mathematical error of neglect". If you would like a pedagogical introduction to the theorem, explaining both its construction and an explanation of what is so fundamental about it, either before or after you continue reading the present text, I suggest an examination of my book [7], Chapter 2.10, pages 99-113.

The remainder of this Section presents an analysis of the implications of theoretical quantum probabilities for the imagined results of the now classic gedankenexperiment on a pair of electrons propelled toward the Stern-Gerlach magnets of Alice and Bob. It covers a description and a formalization of QM-motivated probability assertions appropriate to the several problems posed, and displays computational results that identify the bounding implications for other probabilities about which the theory is silent. Although quantum theory says nothing precise about the gedanken results, the FTP places definitive restrictions on what would cohere with what quantum theory does say.

Section 5.1 is simply discursive, describing conversationally the setup of the linear programming problems relevant to our discussion. It does not dwell on explicit formal definitions of all notation, but rather proceeds straightforwardly with discussion using vocabulary that is standard in LP methods. Section 5.2 
presents formal algebraic detail of the quantities and constraints involved in these LP problems. Section 5.3 then displays numerical results that portray the polytope of probability vectors representing the coherent content of recognized quantum theory as it is relevant to these matters.

\subsection{A Linear Programming Problem Identifying QM Probabilities That Recognize Functional Restrictions: A Discursive Introduction}

To begin, we shall presume standard expectations (and probabilities) of quantum theory for the spin-products in the domain experiments 23 , and use a linear programming format to specify bounds on implied probabilities for spin-product observations in the range experiments 1456789 imagined to be concurrent in the thought experiment. Once we are clear on how this works we shall describe how such a set of $\min / \max$ computations would be replicated and concatenated for all twelve functional restriction structures.

Familiar by now with the quantum probability distributions for the outcomes of any real experiment, you should recall that the probabilities for the four possible outcomes of the spin observations,,,+++--+ , and --, can all be identified from the probability for any one of them, say $P_{++}$. For the four quantum probabilities resolve to $P_{++}=P_{--}$along with $P_{+-}=P_{-+}=\frac{1}{2}\left[1-2 P_{++}\right]$. Furthermore, this probability is uniquely related to the expected value of the spin product via the equation $E(A B)=P_{++}-P_{+-}-P_{-+}+P_{--}$, which then equals $4 P_{++}-1$. The probability for a spin-product of -1 resolves to $P[A B=-1]=[1-E(A B)] / 2$.

In the context of any component experiment for which the relative angle between the two magnet orientations is equal to $\theta$, these relations specify $P_{++}(\theta)=\frac{1}{2}\left[1-\cos ^{2}(\theta / 2)\right]$ and $E[A B(\theta)]=1-2 \cos ^{2}(\theta / 2)$. For reference in our computations relevant to Mermin's problem, these spin-product expectations resolve to $E\left[A B \mid \theta=-120^{\circ}\right]=E\left[A B \mid \theta=+120^{\circ}\right]=0.5$ and $E\left[A B \mid \theta=0^{\circ}\right]=-1$.

Correspondingly,

$P\left[A B=-1 \mid \theta=-120^{\circ}\right]=P\left[A B=-1 \mid \theta=+120^{\circ}\right]=0.25$, and
$P\left[A B=-1 \mid \theta=0^{\circ}\right]=1$.

Here is the problem, stated directly in conversational English, presuming familiarity with all algebraic notation and detail. We are to investigate the QM-motivated probability specifications for the 4 possible observation vectors of nine spin-products observed by Alice and Bob in a $3 \times 3$ paired-angle-thought-experiment on the same pair of electrons. Each of these vectors specifies an array of values for all nine components of the spin-product gedanken vector we shall call

$$
\boldsymbol{G}_{9} \equiv\left(A_{n} B_{n}, A_{n} B_{z}, A_{n} B_{p}, A_{z} B_{n}, A_{z} B_{z}, A_{z} B_{p}, A_{p} B_{n}, A_{p} B_{z}, A_{p} B_{p}\right)^{\mathrm{T}}
$$

These are the quantities that are crucial to the specifications of quantum theory. A complete list of their possible gedanken observations constitutes the four columns of the left half of the middle partition of the realm matrix we created in Section 3.2, and designated as $\boldsymbol{R}_{9,4}$. Only four of the nine rows of this 
matrix are distinct, the other five being repetitions.

Among these possible 9-dimensional vectors of spin-products, there are only two dimensions of free observations, on account of the functional restrictions embedded within them. For example, the observations at the magnet configurations numbered 2, 3 would functionally identify the results at configurations 1,4 , 5, 6, 7, 8, 9 according to quantum theoretic specifications enhanced by Einstein's presumed principle of local realism. However, this functional relation is evidently non-linear, for the rank of the realm matrix of nine spin-product possibilities is 4 . This rank corresponds to any four distinct rows of $\boldsymbol{R}_{9,4}$. Thus, the specification of quantum expectations pertinent to the domain configurations of rows 2 and 3 would place only polytopic bounds on the cohering probabilities for the other spin-products they imply.

If we were to specify a complete distribution of probabilities over the four possible spin-product outcome components of the function domain, $\{-1,+1\}^{2}$, our problem would be over. However, quantum theory explicitly disavows an assertion of a complete distribution vector $\boldsymbol{q}_{4}$ over these possibilities, for this would entail a specification of joint probabilities for the results of non-commuting Hermitian operators on the state space of the electron pair. While quantum theory specifies precise probabilities for the two possible values of the spin-product observation $A_{d} B_{d}$ at any experimental paired angle setting of Stern-Gerlach magnets, it explicitly says nothing about the joint outcomes of the spin-products observed at both settings 2 and 3, for example. Nonetheless, quantum theory does specify explicitly cohering probabilities regarding the outcomes of the spin-product experiment at each of the configurations 2 and 3 separately. Observations at this pair of settings constitute the domain of the function we have designated as $23 \rightarrow 1456789$.

An aside of detail should clarify the preceding disavowal. While QM theory would clearly specify probabilities such as $P\left[\left(A_{n}=1\right)\left(B_{z}=-1\right)\right]$ and $P\left[\left(A_{n}=1\right)\left(B_{p}=+1\right)\right]$ for example, deriving from the expectations $E\left(A_{n} B_{z}\right)$ and $E\left(A_{n} B_{p}\right)$, it explicitly disavows assertions of the form $P\left[\left(A_{n}=1\right)\left(B_{z}=-1\right)\left(A_{n}=1\right)\left(B_{p}=+1\right)\right]$. The former two assertions each specify a standard probabilistic assertion of quantum theory relative to a pair of spin observations at a specific paired angle setting; whereas the latter assertion would entail a claim about joint observations of spins at both $B_{z}$ and $B_{p}$, observations represented by non-commuting Hermitian operators. However, a joint probability of this sort would be required in order to specify precise values for components of the vector $\boldsymbol{q}_{4}$, flaunting this abstemious honesty. Quantum theory does not provide for a complete distribution of probabilities for the four possible spin-product components of $\{-1,+1\}^{2}$, neither at the domain pairing 23 , nor any other pair of domain variables among the twelve functions embedded in the observation realm.

Suppose then that we entertain the cohering expectations of quantum theory pertinent to the isolated domain products 2 and 3 . What would these imply for the cohering expectations of spin-products $1,4,5,6,7,8$, and 9 determined in 
their function range? Based on the associated rows of the realm matrix for the spin products, these expectations would place two linear restrictions on the components of any prospective gedankenvector $\boldsymbol{q}_{4}$. Along with the constraint that their components are all non-negative and sum to 1 (unity), a linear programming routine would identify a pair of solution vectors $\boldsymbol{q}_{4 \min }$ and $\boldsymbol{q}_{4 \max }$ that produce the extreme feasible values of the objective functions $E\left(A_{d} B_{d}\right)$ for any range orientation pairing, subject to the quantum theoretical linear constraints on spin-products 2 and 3. (These are in addition to the requirement that $E(A B)=-1$ for the spin-products of orientation pairs 1,5 , and 9 , a condition specified by quantum theory which underlies the entire problem.) The linear coefficients of the objective function can be identified from appropriate rows of the realm matrix.

As it turns out, with expectations specified for any pair of two domain variables, there is only one range variable whose extreme cohering expectations we shall need to investigate. Remember that considerations of symmetry in the problem imply the equality of the spin products $A_{n} B_{z}=A_{z} B_{n}, A_{n} B_{p}=A_{p} B_{n}$, and $A_{z} B_{p}=A_{p} B_{z}$. These correspond to the identity of rows 2 and 4, 3 and 7, and 6 and 8 in the realm matrix $\boldsymbol{R}_{9,4}$. Thus, with expectations settled at -1 for the negative spin-products at orientations 1,5 , and 9 , asserting quantum theoretic expectations at orientations 2 and 3 would imply the same expectations at orientations 4 and 7 as well. This would leave only spin-product expectations for orientations 6 and 8 to be investigated, and these must be identical. As a result, a single pair of min/max linear programming problems would identify the bounds on the entire cohering expectation vector. This is what we shall formalize now.

The paired solution vectors $\boldsymbol{q}_{4 \min }$ and $\boldsymbol{q}_{4 \max }$ contain the information that puts bounds on the general problem solution we seek: to identify the extreme vectors $\boldsymbol{q}_{4}$ that satisfy all QM-motivated probability specifications relevant to the gedankenexperiment and also support appropriate cohering range expectations. We will need to determine this pair of solution vectors for the $\mathrm{min} / \mathrm{max} \mathrm{LP}$ problems appropriate to each of the twelve function domains we have identified. It would be the entire resulting space of vectors that represents the implications of quantum theory pertinent to the magnetic spin-product gedankenexperiment on an electron pair.

Before presenting the numerical results of these computations, let's examine a formal identification of the quantity vectors that play the central roles in the linear restrictions of the several linear programming problems we have delineated.

\subsection{Algebraic Representation of the LP Constraints}

Our goal is identify the prognostications of quantum theory regarding a gedankenexperiment: Mermin's physics problem of two electrons engaging the nine component $3 \times 3$ design of Stern-Gerlach magnets at the stations of Alice and Bob.

Let $\boldsymbol{G}_{9}=\left(A_{n} B_{n}, A_{n} B_{z}, A_{n} B_{p}, A_{z} B_{n}, A_{z} B_{z}, A_{z} B_{p}, A_{p} B_{n}, A_{p} B_{z}, A_{p} B_{p}\right)^{\mathrm{T}}$ denote the 
column vector of imagined spin-product outcomes of the nine experiments on a single pair of electrons.

Let $\boldsymbol{R}_{9,4} \equiv \boldsymbol{R}\left(\boldsymbol{G}_{9}\right)$ designate the realm matrix of possibilities for the gedankenobservation vector $\boldsymbol{G}_{9}$. We have already displayed it as the left half of the middle matrix partition of the large realm matrix we constructed in Section 3.2. We shall refer to individual rows of this matrix by using the denotation $\boldsymbol{r}_{i}$. for values of the row numbers $i=1, \cdots, 9$, and to individual columns of this matrix by $\boldsymbol{r}_{\cdot j}$ for values of the column numbers $j=1,2,3,4$.

The bold vector $\mathbf{1}_{4}$ denotes a row vector of four 1's.

Furthermore, let the column vector $\boldsymbol{b}_{9}$ denote the numerical values of the quantum theoretic expectations for the components of $\boldsymbol{G}_{9}$ when they each designate the outcome of a single real experiment on a pair of electrons at a specific angle pairing of the $S-G$ magnets.

Finally, let $\boldsymbol{Q}_{4}$ denote the column vector of events whose components identify whether the observation vector $\boldsymbol{G}_{9}$ would happen to equal the various columns of $\boldsymbol{R}_{9,4}$. That is, the component $Q_{j}=\left(\boldsymbol{G}_{9}=\boldsymbol{r}_{. j}\right)$. Each of these four events equals 1 or 0 , and only one of them equals 1 .

Notice that whereas quantum theory specifies expectations for the components of $\boldsymbol{G}_{9}$ when each is entertained as the spin-product of a unique pair of electrons in individual experiments standing alone, it does not specify expectations for the components of $\boldsymbol{Q}_{4}$. For these events identify the joint outcomes of several incompatible experimental observations. However, the linear programming problems we now address codify restrictions on the space of such expectation vectors that would cohere with what quantum theory does say about the domain experiments individually.

To begin, we should formalize the linear programming investigations required by the spin-product function $23 \rightarrow 1456789$ which we have discussed informally in the details of the preceding subsection:

Find the vectors $\boldsymbol{q}_{4 \min }$ and $\boldsymbol{q}_{4 \max }$ that yield the minimum and the maximum values of $\boldsymbol{r}_{6} . q_{4}$ subject to the conditions that

$$
\left(\begin{array}{l}
\boldsymbol{r}_{2} \\
\boldsymbol{r}_{3} \cdot \\
\mathbf{1}_{4}
\end{array}\right) \boldsymbol{q}_{4}=\left(\begin{array}{c}
b_{2} \\
b_{3} \\
1
\end{array}\right),
$$

where each component $q_{i} \geq 0$.

We shall denote these two solution vectors by $\boldsymbol{q}_{4 \min 23.6}$ and $\boldsymbol{q}_{4 \max 23.6}$. For once we determine them, we shall need to repeat such computational LP searches so to determine extreme vectors appropriate to the other eleven spin-product functions that govern possibilities for the spin-product vector $\boldsymbol{G}_{9}$ as well. Formally, this would amount merely to changing the coefficient vectors $\boldsymbol{r}_{2}$. and $\boldsymbol{r}_{3}$. in the LP domain constraints, and changing the objective function coefficients $\boldsymbol{r}_{6}$. accordingly so to represent the range variable whose expectation bounds we search. In principle, there could be 24 such extreme solution vectors. However, on account of duplications among the row vectors of $\boldsymbol{R}_{9,4}$, 
there are only three distinct LP problem pairs among these, and six distinct solution vectors. It should be evident, for example, that $\boldsymbol{q}_{4 \min 23.6}=\boldsymbol{q}_{4 \min 47.8}$ and $\boldsymbol{q}_{4 \max 23.6}=\boldsymbol{q}_{4 \max 47.8}$. The LP problems that they resolve are numerically identical.

It is a feature of coherent probability structures that any convex combination of coherent expectation assertions is also coherent. It is the convex hull of all the six extreme expectation vectors that represents the quantum theoretic prognostications for the gedankenexperiment.

\subsection{Computational Results}

The six solution vectors that resolve the three distinct pairs of LP problems involved in this investigation are displayed as $4 \times 1$ columns in the top section of Table 3. The notational subscripts in their column headings specify the form of the LP problems from which they derived. Although they were designed to identify extreme values of expected spin-products at specific magnet orientations, the probabilities underlying each of these extreme solution vectors would specify cohering expectation values for every one of the spin-products that result from a "run" of the gedankenexperiment at all nine relative angle configurations. These implied nine-vectors of expected spin-products are printed immediately below the solution vectors in the Table to which they correspond. The rank of the matrix of solution vector columns is 4 .

Table 3. Extreme spin-product expectations for unique LP solutions.

\begin{tabular}{|c|c|c|c|c|c|c|c|c|}
\hline LP solutions & $\boldsymbol{q}_{4 \min 23 \cdot 6}$ & $\boldsymbol{q}_{4 \min 26.3}$ & $\boldsymbol{q}_{4 \min 36.2}$ & $\boldsymbol{q}_{4 \max 23.6}$ & $\boldsymbol{q}_{4 \max 26 \cdot 3}$ & $\boldsymbol{q}_{4 \max 36.2}$ & & \\
\hline$q_{1}$ & 0.25 & 0.25 & 0.25 & 0 & 0 & 0 & & \\
\hline$q_{2}$ & 0 & 0 & 0.75 & 0.25 & 0.25 & 0.5 & & \\
\hline$q_{3}$ & 0 & 0.75 & 0 & 0.25 & 0.5 & 0.25 & & \\
\hline$q_{4}$ & 0.75 & 0 & 0 & 0.5 & 0.25 & 0.25 & & \\
\hline$E$ (spinprod) & & & & & & & $\mathrm{QM}$ & Sim \\
\hline$E\left(A_{n} B_{n}\right)_{1}$ & -1 & -1 & -1 & -1 & -1 & -1 & -1 & -1 \\
\hline$E\left(A_{n} B_{z}\right)_{2}$ & 0.5 & 0.5 & -1 & 0.5 & 0.5 & 0 & 0.5 & 0.25 \\
\hline$E\left(A_{n} B_{p}\right)_{3}$ & 0.5 & -1 & 0.5 & 0.5 & 0 & 0.5 & 0.5 & 0.25 \\
\hline$E\left(A_{z} B_{n}\right)_{4}$ & 0.5 & 0.5 & -1 & 0.5 & 0.5 & 0 & 0.5 & 0.25 \\
\hline$E\left(A_{z} B_{z}\right)_{5}$ & -1 & -1 & -1 & -1 & -1 & -1 & -1 & -1 \\
\hline$E\left(A_{z} B_{p}\right)_{6}$ & -1 & 0.5 & 0.5 & 0 & 0.5 & 0.5 & 0.5 & 0.25 \\
\hline$E\left(A_{p} B_{n}\right)_{7}$ & 0.5 & -1 & 0.5 & 0.5 & 0 & 0.5 & 0.5 & 0.25 \\
\hline$E\left(A_{p} B_{z}\right)_{8}$ & -1 & 0.5 & 0.5 & 0 & 0.5 & 0.5 & 0.5 & 0.25 \\
\hline$E\left(A_{p} B_{p}\right)_{9}$ & -1 & -1 & -1 & -1 & -1 & -1 & -1 & -1 \\
\hline
\end{tabular}


To the right of the six $E$ (spinprod) vectors in the Table appear two additional column vectors for purposes of comparison. The column headed by $\mathrm{QM}$ exhibits the standard expectations of quantum mechanics for the spin-product to be observed in an actual experiment at any single one of the various paired angle configurations. The final additional column headed by Sim exhibits expectations for spin-products as 0.25 corresponding to those proportions of differing spin observations $(-+)$ or $(+-)$ that were generated in our simulated gedankenexperiment in Section 4.1, with those proportions rounded to 0.375 . The simulations, remember, relied upon quantum-theory-motivated probabilities to generate the emergence of spin-products at each of the domain configurations, and then relied on the functional relations to yield the other seven dimensional components accordingly. The simulated behaviour of each electron pair involved its engaging the magnet orientations at detection stations in all nine of their experimental paired orientations. This is the situation that provoked Mermin's rejection of the message-encoded balls explanation.

The glory of Table 3 is that its columns specify the extreme points of a bounding polytope of expectation vectors supported by quantum theory as it would be relevant to the spin-products of a gedankenexperiment on a single pair of electrons at all nine of the possible orientation pairings. The fundamental theorem of probability does not identify a specific vector of expectations for spin-products at every one of the nine paired magnet orientations. Neither do the prescriptions of quantum theory, which avoid precise assertions regarding the joint outcomes of non-commuting measurements. Rather, they specify a space of such allowable expectation vectors which cohere with the assertions about real experiments that quantum theory actually is endowed to assess. Based on the QM motivated probabilities that constrained the several LP computations, the conclusion to this exercise is that the sought-for vector of gedanken expectations needs merely sit somewhere within the convex hull of the $\mathrm{E}$ (spinprod) vectors appearing as the first six columns in the lower half of the Table.

The rank of the matrix of 9-D expectation vectors appearing in Table 3 is only four. These four dimensions are spanned by the rows 1,2,3, and 6 of the Table. The other rows are repeats of these, so we could have listed four different rows, but the result would be the same. In order to discuss these results in the same terms with which Professor Mermin assessed the behaviour of his machine, we shall transform these expectations into the probabilities they imply for negative spin values in Table 4. Expressed as a function of $\theta$, this linear transform is $P[A B(\theta)=-1]=\{1-E[A B(\theta)]\} / 2$ for each relative angle between the magnet orientations.

One of the features of these computational results is that we can now make sense of the simulation results we generated in Table 2. These, remember, defied Professor Mermin's claims regarding results that would obtain in such (impossible) experimentation on a single electron pair. The simulated counts of negative spin-products shown in that Table yielded proportions on the order of 0.375 for 
six of the Stern-Gerlach orientation pairings. These differ markedly from the Mermin proclamation that proportions of negative spin-products would hardly differ from 0.25 at these settings, but they do exhibit the order of magnitude that he found upsetting. As a marginal statement in the context of the gedankenexperiment, quantum theory specifies only $P\left(A_{n} B_{z}=-1\right) \in[0.25,1]$, for example, which can be seen by reading along the rows of Table 4 . But we can be more specific in understanding the three such marginal assessments of probability regarding $A_{n} B_{z}, A_{n} B_{p}$, and $A_{z} B_{p}$. Since the top row of the Table is constant at the value of 1 , we can recognize that the convex hull enclosing the first six column vectors of this matrix constitutes a 3 -D polytope within a hyperplane in the 4-D space. It is displayed in Figure 2 as a tetrahedron that has lost one of its tips.

Table 4. Probabilities for negative spin-products along extreme solution vectors.

\begin{tabular}{ccccccccc}
\hline LP solutions & $\boldsymbol{q}_{4 \min 23.6}$ & $\boldsymbol{q}_{4 \min 26.3}$ & $\boldsymbol{q}_{4 \min 36.2}$ & $\boldsymbol{q}_{4 \max 23.6}$ & $\boldsymbol{q}_{4 \max 26.3}$ & $\boldsymbol{q}_{4 \max 36.2}$ & QM & Sim \\
\hline$P\left(A_{n} B_{n}=-1\right)_{1}$ & 1 & 1 & 1 & 1 & 1 & 1 & 1 & 1 \\
$P\left(A_{n} B_{z}=-1\right)_{2}$ & 0.25 & 0.25 & 1 & 0.25 & 0.25 & 0.5 & 0.25 & 0.375 \\
$P\left(A_{n} B_{p}=-1\right)_{3}$ & 0.25 & 1 & 0.25 & 0.25 & 0.5 & 0.25 & 0.25 & 0.375 \\
$P\left(A_{z} B_{p}=-1\right)_{6}$ & 1 & 0.25 & 0.25 & 0.5 & 0.25 & 0.25 & 0.25 & 0.375 \\
\hline
\end{tabular}

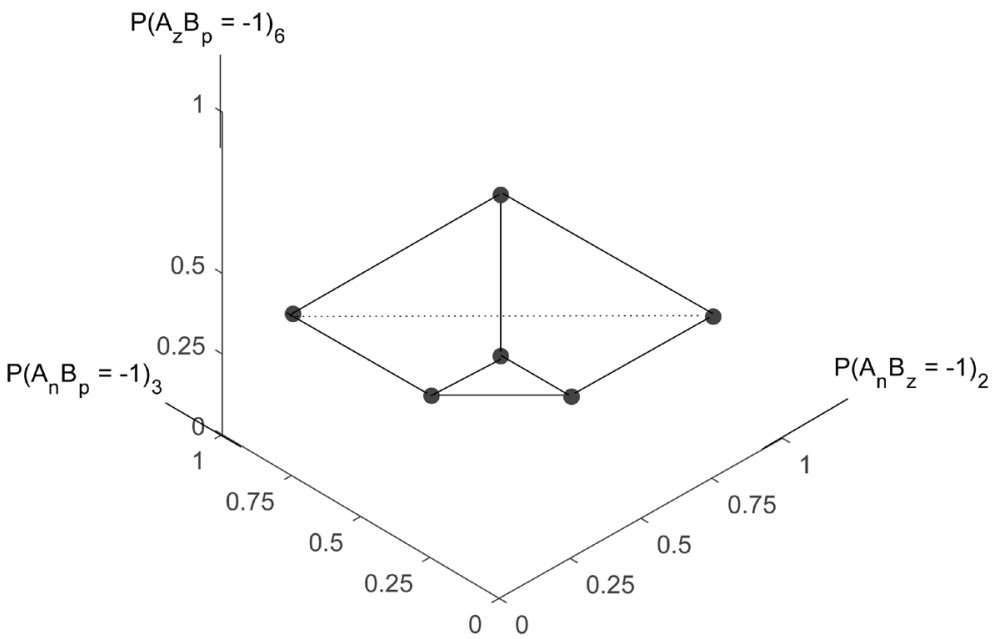

Figure 2. The three dimensional polygon constituting the convex hull of probabilities for negative spin products in a gedankenexperiment when the Stern-Gerlach magnet directions are not set identically. Professor Mermin's proclaimed point of probabilities in these three dimensions, $(0.25,0.25,0.25)$, is exterior to this polytope, while the simulation vector of probabilities $(0.375,0.375,0.375)$ is a point well within the hull as a convex combination of its vertices.

Regarding the first six columns of Table 4 as vertices of a quantum theoretical probability polytope for negative spin-products, we can determine that the appended final column vector headed by Sim is located within their convex hull. Algebraically, it is equal to the convex combination of those columns with con- 
vexity coefficients $(1 / 6,0,1 / 12,0,1 / 2,1 / 4)$. In contrast, the appended column headed by QM does not lie in this convex hull. It cannot be expressed as a convex combination of the vertex vectors. To proclaim it as representing the prescriptions of quantum mechanics for the outcome of the gedankenexperiment would be incoherent. The accompanying relegation of Einstein's principle of local realism amounts to nonsense. When the operation of Professor Mermin's machine is applied to the gedankenexperiment, the crude vector of quantum probabilities representing his provocative claims lies outside of the convex hull of probability vectors that are supported by the results of quantum theory. His assertions and his concerns derive from a mathematical error of neglect, similar in structure to the error we have found in the claims of Aspect/Bell.

\section{A Mystery Exposed}

In challenging the purported mysteries of quantum physics, we have now been through an exercise of tedious mathematics and computation. It is time to conclude with an overview of what we have learned. I will be brief and frank in concluding. An extensive discussion of issues in quantum theory that impinge on the foundations of probability will require a presentation of its own [8].

There is nothing wrong with Professor Mermin's machine, and furthermore there is nothing mysterious about it. The machine quite accurately portrays the probabilistic structure of the current propositions of quantum theory regarding the experimental observation of magnetic spins of paired electrons as they pass angled Stern-Gerlach magnets at two distantly separated detection stations. To be explicitly precise, the machine is designed to exemplify the structure of theoretical and empirical results from sequential observations of distinct electron pairs ejected to any one of nine different paired experimental settings, similar in design. Each of the paired dial settings on the two machines constitutes a different type of experiment. At three of the paired angle magnet orientations when the two settings are identical, every observation of the spin-product equals -1 . The spins $(A, B)$ observed at the two stations are recorded as either $(-1,+1)$ or $(+1,-1)$. At any one of the six other paired settings in which the magnet orientations differ, long sequences of observations yield spin-products equal to -1 in $1 / 4$ of the runs, and +1 in $3 / 4$ of the runs. For any individual unique experiment designed with such a structure, quantum theory asserts only the probability of $1 / 4$ for the negative spin-product. The quantum probability for the electron spin behaviour at station A depends on the orientation of the magnet at station $\mathrm{B}$ as well as that at station $\mathrm{A}$.

Many regard this result to be mysterious in itself, involving what Einstein had referred to as "spooky action at a distance". The source of this attitude derives from the imagination that the probabilistic behaviour of quantum activity corresponds to a feature of randomness inherent in the particle structure. Nature at its fundamental base is considered to be random, governed by recognizable probability distributions, derivable by theoretical acumen and tamed at classical 
scales of mass phenomena by the laws of large numbers. It is this conception of the matter to which Einstein objected. He proposed with EPR that the stochastic aspect of quantum theoretic results derives rather from the incompleteness of the theory and from our uncertainty regarding the influence of unknown and unobserved "supplementary variables" pertinent to the conditions of any specific experimental run. This proposal would relegate "the mystery" of quantum results to the same category involved in common mysteries of activity at classical scales of magnitude, such as "where did I leave my keys?". Maybe here, maybe there. It is the codification of symmetries in our uncertainty regarding conditions of the experimental problem that yields probabilistic prescriptions regarding the quantum mechanics. Conditional probabilities for the outcome of one quantity that vary with the outcome of a conditioning event are a standard feature of distributions that represent exchangeable (symmetric) judgments. There is no implication that the conditioning event has actually been observed. A conditional probability will be specified conditioned on the negated event as well. Both, together, characterise an asserted quantum probability distribution for the possible paired results of the experiment.

When regarded as properties of the particles themselves, the quantum probabilities of Mermin's machine do seem to pose the mysterious question of how the probabilistic activity of the electron at station A can depend on the magnet angle (dial) setting at station $B$ if there is no way for the status of the setting at $B$ to be communicated to station A when an electron (a ball) arrives there. The resolution of this enigma proceeds from recognition that probabilities are not properties of particles at all, but rather formally assessed representations of our considered uncertainty about observable quantities. For now, I shall focus my conclusion here on what the professor proclaims as a challenge to this point of view. It underlies a mistaken attitude that is held virtually universally among quantum theorists today: the defiance of Bell's inequality in a gedankenexperiment on a single pair of electrons at all nine experimental settings defies not only the reasonability of local realism at the quantum scale and the proposition of supplementary variables pertinent to quantum behaviour, but the uncertainty interpretation of probabilities itself in accounting for the evidence of quantum experiments. Indeed it is a common misconception that the defiance of the inequality arises in quantum mechanical assessment of spin product expectations for any electron pairs whatsoever, gedanken or not.

Professor Mermin models the action of supplementary variables by encoded designations of colour schemes on the balls ejected toward the stations. He proposes this as a model of most any supplementary variables explanation of the quantum experiment, on the same metaphorical order as the machine with balls models the observation of electron spin behaviour. Such a proposal could be fair enough, though one might quibble with its adequacy for representing the substance of the supplementary variables viewpoint. Nonetheless, his analysis of the activity of an encoded pair of balls when they arrive at all nine dial settings generates what he considers to be an even deeper mystery. Although the scheme 
surely ensures matching light signals when the dials are set identically, he motivates the proportion of matching lights (spin-products equal to -1) deriving from such a scenario as equal to more than $1 / 3$. It is this result, which he proposes as an instance of the supposed defiance of Bell's inequality, that is seen to constitute the mystery of quantum behaviour: apparently no supplementary features of the experimental situation can account for the known behaviour of quantum experiments.

It is this result that is just plain wrong. Examining the real quantum experiment which we are coaxed to ignore, we have found embedded within the corresponding thought experiment a surprising feature that has long been unnoticed. Subjecting each pair of electrons to spin-detection at all nine of the paired angle settings would engender an array of restrictive functional relations among the nine observed spin results. The professor neglects these symmetric functional relations mapping $\{-1,+1\}^{2}$ into $\{-1,+1\}^{7}$ in his analysis of the situation. His claims regarding the machine behaviour yielding matching lights in $1 / 4$ of such gedanken observations when the switches differ are blatantly false. They rely on the possibility that any string of nine-tuples deriving from the cartesian product $\{-1,+1\}^{6}$ of spin possibilities could designate the outcome of such a thought experiment. We have seen otherwise ... that many such strings of spin-products are impossible. The space of possibilities derives rather from the cartesian structure $\{-1,+1\}^{2}$, replicated in several different pairs of angled magnet orientations. The four possible results of each domain pair of spin observations is mapped into restrictive completions within the space of $\{-1,+1\}^{7}$. Moreover, we have used computational procedures of linear programming to identify precisely the polytope of probabilistic assertions regarding the outcomes of the gedankenexperiment that represent the honest claims of quantum theory relevant to this matter.

The probabilities for matching lights proclaimed by Mermin are representable by a nine-tuple vector that does not lie within the convex hull of the coherent vectors supported by quantum theory. We have created a Monte-Carlo simulation of results of a scenario which is both wholly consistent with quantum theory and also respects the restrictive symmetric functional relations that govern the structure of the experiment. It generates proportions of matching lights on the order of 0.375 , precisely on the order of magnitude that the professor would have us suspect on account of his error of neglect. This vector does reside within the convex hull of extreme gedanken probabilities required by coherency. But there is still more to say about this!

One of the most famous features of quantum theory, known widely by name to the general public, is the relevance of Heisenberg's uncertainty principle. Under the guise of that name, the principle concerns physical experiments with electrons that attempt to measure both the position and the velocity of an electron at a point in time. What the principle recognizes is that it is impossible to make such a joint measurement of both of these characteristics of the electron at the same time. We can make a measurement of one or the other, but not both. 
Technically, the quantum theory identifies this impossibility by the characterization of the two measurements of the quantum state via Hermitian operators that do not commute. Quantum theory can specify probability distributions for possible values of either of these measurements on an electron. However, it cannot and does not provide an assessment of a joint probability for the observation of both measurements. For such an operation is impossible. It is the incommensurability of measurements characterised by matrix operators which do not commute that is the general form of the uncertainty principle of theoretical quantum mechanics.

The incommensurability of simultaneous observation of paired electron spins at several different paired magnet orientations in a gedankenexperiment belies the professor's claims about his machine in this context. Quantum theory does not identify a joint probability distribution for the results of all nine of them. This limitation has long been recognized since the early insights of Fine [9] [10] and subsequently in challenges by Hess and Phillip [11], among others. These have been regularly rebuffed in mainstream literature which has heralded the rejection of local realism on this account. Nonetheless the message relevant to the incompleteness of quantum theory is clear and is accentuated by the deliberations I have reported herein. A stirring review by Kupczynski [12] has recently supported a revived reconsideration of the widely acclaimed rejection of local realism.

Quantum theory does provide precise probabilities for the four possible observations of spin pairs at any single paired orientation, these being,,+++--+ , or -- at the two detection stations. Moreover, it can stipulate probabilities for such outcomes from several distinct experiments on an electron pair at differing magnet angles, realizing albeit that only one of them can be engaged. These are the probabilities used in linear programming routines that identify the restrictions on range probabilities provided by individual assessments of domain probabilities. However, for the joint distribution of all nine measurements it leaves four dimensions of freedom remaining unascertained. The probability distributions of quantum theory for the results of a real experiment on an electron pair at any one of the nine design orientations may not be considered to be a marginal distribution from a joint distribution. There is no joint distribution over these imagined experimental results supported by quantum theory in its current formulation! Any vector within the polytope of feasible joint distributions can be transformed mechanically into a vector of marginal probabilities for the spin products at any magnet orientation pair, but no one of these constitutes a marginal distribution pertinent to all feasibilities.

In particular, the result of our simulated experiment using the probabilistic assertions of quantum theory cannot be presumed to provide a definitive proclamation of quantum mechanics. It is intriguing that it generates coherent probabilistic activity that emulates precisely behaviour of the sort that Professor Mermin had relegated. Yet the implications of the simulation are not decisive. Its generation had embedded into it a formulation of independence among the 
outcomes of any two spin-products observed among the domain arguments of the functions. While feasible in the context of the agnostic stance of the theory relative to incommensurable measurements, this is surely not a requirement of the theory. Furthermore, in the context of the active claims of the theory regarding the entanglement of spin observations at distant detection stations, it ought well be considered suspect by those who might like to think about such things. We are left with the realization that quantum theory provides only a convex polytopic boundary of probabilities for the result of the gedankenexperiment to which Bell's inequality is relevant. Bell's inequality is defied by none of the distributions within the polytope that is entertained by quantum theory as we know it. While we are avowedly still to understand completely the physical details of quantum behaviour, they inhere no mysteries in themselves ... for anyone. More hoojums than boojums.

\section{Acknowledgements}

The University of Canterbury provided computing and research facilities. Thanks to Larry Dennis for helpful discussions, to the managing editor and three reviewers for very helpful comments, and to Paul Brouwers, Steve Gourdie, and Allen Witt for IT service and consultation.

\section{Conflicts of Interest}

The author declares no conflicts of interest regarding the publication of this paper.

\section{References}

[1] Mermin, N.D. (1981) Journal of Philosophy, 78, 397-408. https://doi.org/10.2307/2026482

[2] Mermin, N.D. (1990) Boojums All the Way Through: Communicating Science in a Prosaic Age. Cambridge University Press, Cambridge. https://doi.org/10.1017/CBO9780511608216

[3] Lad, F. (2021) Journal of Modern Physics, 12, 1109-1144. https://doi.org/10.4236/jmp.2021.128067

[4] Lad, F. (2020) Entropy, 22, 19. https://doi.org/10.3390/e22070759

[5] Einstein, A., Podolsky, B. and Rosen, N. (1935) Physical Review, 47, 777-780. https://doi.org/10.1103/PhysRev.47.777

[6] de Finetti, B. (1970) Teoria delle probabilità. Ed. Einaudi, 2 voll., Torino. (English version: Theory of Probability. A. Machi and A. Smith (trs.), Chichester, Wiley, 1974, 1975).

[7] Lad, F. (1996) Operational Subjective Statistical Methods: A Mathematical, Philosophical, and Historical Introduction. John Wiley, New York.

[8] Lad, F. (2021) On Probability and Quantum Physics. Preprint on Researchgate. https://doi.org/10.13140/RG.2.2.21376.87048

[9] Fine, A. (1982) Physics Review Letters, 48, 291-295.

https://doi.org/10.1103/PhysRevLett.48.291 
[10] Fine, A. (1989) Do Correlations Need to Be Explained? In: Cushing, J.T. and McMullin, E., Eds., Philosophical Consequences of Quantum Theory (pp. 175-194). University of Notre Dame Press, Notre Dame, Indiana.

[11] Hess, K. and Philipp, W. (2005) Bell's Theorem: Critique of Proofs with and without Inequalities. AIP Conference Proceedings, 750, 150-157.

https://doi.org/10.1063/1.1874568

[12] Kupczynski, M. (2020) Frontiers in Physics, 8, 273. https://doi.org/10.3389/fphy.2020.00273

[13] Bruno, G. and Gilio, A. (1980) Statistica, 40, 337-344.

[14] Lad, F., Dickey, J.M. and Rahman, M.A. (1990) Statistica, 50, 19-38.

\section{Appendix: The Fundamental Theorem of Probability}

The fundamental theorem of probability (FTP) specifies that when probabilities or expectations for any $N$ quantities whatsoever are assessed with the vector of values $\boldsymbol{p}_{N}$, then bounds on a cohering expectation for any further $(N+1)^{\text {st }}$ quantity can be computed via a linear programming routine. The theorem was first named in de Finetti [6] (Chapter 3.10), though it is as old as his famous lectures at the Institute Henri Poincaré in 1935. It was first presented in linear programming form in the article of Bruno and Gilio [13]. The theorem extends naturally to specify bounds on expectations for general quantities ("previsions" in de Finetti's nomenclature) as presented in the article of Lad, Dickey, and Rahman [14]. It is discussed pedagogically in Lad [7] (Chapter 2.10, pp. 99-113).

Theorem: Suppose the realm matrix $\boldsymbol{R}\left(\boldsymbol{X}_{N+1}\right)$ for the vector of quantities $X_{1}$ through $X_{N+1}$ has $K$ columns. These columns exhaust all possibilities for prospective quantity observations under consideration. Define the vector $\boldsymbol{r}_{N+1}$ as the final row of this realm matrix corresponding to the possibilities for the quantity $X_{N+1}$ as the last component of the observation vector, and the matrix $\boldsymbol{R}_{N, K}$ as the $N$ initial rows of the realm matrix corresponding to the concomitant possibilities for the first $N$ components of $\boldsymbol{X}_{N+1}$. The design of the linear programming routine is to find the column vectors $\boldsymbol{q}_{K}$ for which the linear combination $\boldsymbol{r}_{N+1} \boldsymbol{q}_{K}$ achieves minimum and maximum values subject to the $N$ linear restrictions that $\boldsymbol{R}_{N, K} \boldsymbol{q}_{K}$ equals $\boldsymbol{p}_{N}$, along with the restrictions that the components of $\boldsymbol{q}_{K}$ are non-negative and that they sum to 1. If there is no feasible solution to these problems, then the assertion of the $N$ expectations that have been presumed is incoherent.

Comment: The linear restrictions on $\boldsymbol{q}_{K}$ ensure that as long as $E\left(X_{N+1}\right)$ is within the extremes of $\boldsymbol{r}_{N+1} \boldsymbol{q}_{K}$ determined by the theorem, the expectation of the full vector $E\left(\boldsymbol{X}_{N+1}\right)$ would then lie within the convex hull of the columns of its realm matrix. This is the general condition of coherency. 